\title{
Developmental regulation of albumin and $\alpha$-fetoprotein gene expression in the rat
}

Louis Muglia and Joseph Locker*+

Departments of Pathology, Biochemistry, and Biophysics and Theoretical Biology, University of Chicago, Chicago IL 60637, USA

Received 29 May 1984; Revised and Accepted 14 August 1984

\section{ABSTRACT}

Albumin and AFP comprise a developmentally regulated gene family expressed predominantly in liver and yolk sac. In this study, we have analyzed the developmental changes in the levels of transcripts in liver and yolk sac, and the methylation of the genes in yolk sac. We have previously analyzed the gene methylation in developing liver and non-expressing tissues (Kunnath and Locker, 1983, EMBO J., 2, 317-324). From these analyses we can distinguish 3 active modes of coordinated expression. The adult liver synthesizes albumin, the yolk sac synthesizes AFP, and the fetal liver synthesizes both. In the yolk sac, the relatively inactive albumin gene is hypermethylated, while the active AFP gene is undermethylated. Our data suggest that the genes are activated simultaneously but that subsequent enhancement is specific for tissue and developmental stage.

\section{INTRODUCTION}

Albumin and $\alpha$-fetoprotein (AFP) comprise a family of closely related serum proteins that show changing patterns of tissue-specific synthesis during development. AFP, synthesized primarily in the liver and yolk sac, is the most abundant serum protein in the fetus but almost undetectable in the adult. Albumin, synthesized primarily in the liver, is the most abundant serum protein after birth. Because of the considerable structural homology between the two proteins (1-3), the functional differences that distinguish AFP from albumin in fetal homeostasis remain unclear. In the mouse, the two genes are adjacent to one another on chromosome 5 (4), and the developmental regulation implies a gene switching process analogous to the $\gamma-\beta$ globin switch of developing erythroid cells.

In our laboratory, we previously characterized developmental changes in the DNA methylation (5) and chromatin structure (6) of these genes in the liver. In the present study we have defined the associated changes in mRNA levels. In addition, we have now characterized albumin and AFP gene expression in the fetal yolk sac. Our study of the rat, which confirms the observations of Tilghman and Belayew (7) in mouse liver, and sellem et al. 
(8) in the rat, extends the analysis to earlier gestational stages when the genes are first activated, and to DNA methylation.

The rat shows at least three distinct modes of coordinated gene activity: an adult liver mode, in which albumin mRNA is synthesized at maximal levels and AFP mRNA is made at barely detectable levels; a fetal liver mode, in which both mRNAs are synthesized at maximal levels; and a yolk sac mode, in which AFP mRNA is synthesized at maximal levels and albumin mRNA is barely detectable. These modes of expression are primarily controlled at the level of transcription, demonstrated by the measurement of relative levels of transcription by Tilghman and Belayew (7) and the evidence for the lack of post-transcriptional control of these genes presented by Nahon et al. (9). Activation of these modes of synthesis, and switching between modes, are both slow processes that take place over several days during development.

\section{MATERIALS AND METHODS}

Animals. Timed pregnant female and other adult Sprague-Dawley rats were obtained from the Holtzman Company, Madison, WI. Gestational age of the rats was determined from data provided by the supplier and from developmental landmarks (10).

DNA clones. Rat albumin cDNA clones PRSA13, pRSA57, and pRSA510 (11, 12), and AFP CDNA clones PRAF65 and PRAF87 (2), were kindly provided by Thomas Sargent, California Institute of Technology, Pasadena, CA.

Purification of RNA. Cytoplasmic RNA was purified by sodium dodecyl sulfate (SDS)-chloroform extraction of post-nuclear supernatants as described in Muglia and Locker (13). The extraction and purification procedures were monitored by dot blot analysis to assure total extraction of all hybridizable RNA. Dot blots demonstrated that recovery was quantitative if a dilute post-nuclear supernatant (i.e., $20 \mathrm{ml}$ homogenization buffer per gm tissue) was extracted several times with SDS-phenol:chloroform. RNA purification from whole organs, by homogenization of tissue either in SDS-phenol:chloroform (14), or in guanidium isothiocynate (15), gave high-quality RNA but reduced recovery of albumin and AFP mRNA. This decreased recovery was probably due to the high viscosity and high protein concentration of these extracts.

Analysis of RNA. The concentration of purified RNA was measured with the orcinol reaction (16). The quality of RNA was assessed from stained agarose-urea gels (17). Only preparations of undegraded RNA, in which the ratio of $28 \mathrm{~S}$ to $18 \mathrm{~S}$ RNA was about $2: 1$, were further studied. For 
hybridization analysis, each agarose-urea gel lane contained $10 \mu \mathrm{g}$ RNA, which was dissolved in $10 \mu 1$ of 758 formamide, $10 \mathrm{mM}$ Tris, $\mathrm{pH} 7.6,1 \mathrm{mM}$ EDTA, $100 \mu \mathrm{g} / \mathrm{ml}$ aurin tricarboxylic acid. The gels were blotted to nylon membranes (Ceriatran, D and I Filter Corp., Woodburn, MA) as described in Muglia and Locker (13).

$R_{0} t$ Curves. Excess RNA-CDNA hybridization analysis was carried out according to Tse et al. (18). For this analysis, plasnid DNA was nick-translated (19). The cloned inserts were excised with PstI and purified from an agarose gel (20). Hybridization was carried out at DNA concentrations of 0.8 and $4.0 \mathrm{ng} / \mathrm{ml}$, in the presence of a 300-1000-fold excess of hybridizable RNA. Control analysis indicated insignificant self-renaturation of the DNA during the hybridization.

Analysis of DNA methylation. Average methylation of yolk sac DNA was quantitated by densitometric analysis of HpaII and MspI digests of high-molecular-weight DNA, resolved on agarose gels, as described in Kunnath and Locker (21). We controlled for complete digestion by including DNA in control samples and by examination of the mitochondrial. DNA bands in the experimental digests (21). Specific methylation of the albumin and AFP genes was analyzed by Southern hybridization (2.2) to HpaII and MspI digests (5).

Other methods. Purification of high-molecular-weight DNA, restriction enzyme digestion, gel electrophoresis, DNA blotting, and nucleic acid hybridization were carried out as described in Kunnath and Locker (25). Hybridization used 0.2 to $1 \times 10^{6}$ counts/ml of nick-translated $1^{32}$ P]-CDNA. SDS-acrylamiae protein gels were run according to Hames (24).

RESUITS

RNA Transfer Hybridizations. Cytoplasmic RNA was extracted from yolk sacs of $10,12,14,16$, and 18 days' gestation and from livers of the same ages. The liver could not be isolated in 10-day embryos, so the specimen was taken from the red region of the foregut from which the liver arises. We also extracted RNA from 1-day-old neonatal (equivalant to 22 days' gestation) and adult livers. $10 \mu \mathrm{g}$ aliquots of each RNA preparation were resolved on agarose-urea gels, blotted to nylon membranes, and hybridized to albumin and AFP CDNA probes. Representative experiments are illustrated in Figure 1 (yolk sac) and Figure 2 (liver). In all cases, the RNA hybridized to the expected band of mRNA and also to a blur of degraded mRNA extending downward from the band. The ratio of band to blur was highly 

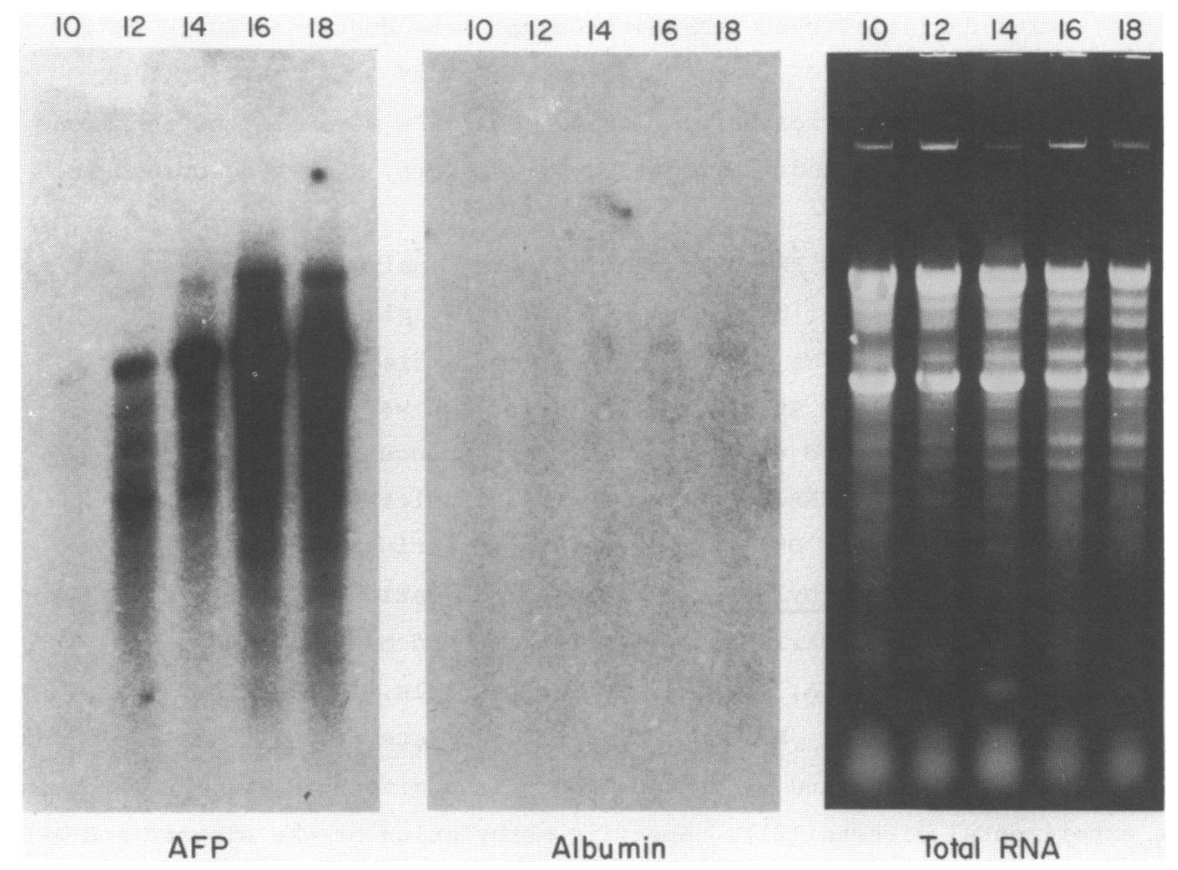

Figure 1. Detection of albumin and AFP mRNA in yolk sac RNA. $10 \mu g$ of RNA from 10-, 12-, 14-, 16-, and 18-day yolk sacs was resolved on $1.5 \%$ agarose-6M urea gels, blotted to nylon membranes, and hybridized with AFP or albumin CDNA probes. The left panel shows hybridization to pRAF87, and the middle panel shows hybridization to pRSA57. The panel on the right shows a stained gel used in one of the blots. Both blots were prepared from matched sets of RNA resolved on the same gel. Hybridizations were carried out in the same bags as the blots in Figure 2. The figure illustrates long exposures to accentuate faint bands, but it should be noted that this exaggerates the relative abundance of degraded mRNA.

consistent for each mRNA, and did not vary from preparation to preparation, even when different extraction methods were used. The stained RNA bands (see Figure 1) showed no evidence of degradation on these gels. We thus hypothesize that the observed degradation occurred in vivo as part of the physiologic turnover of RNA, although actual experimental analysis of this phenomenon is beyond the scope of our study. If poly-A RNA is purified from such preparations, the blur is almost entirely removed from the poly-A-containing fraction so that these observations cannot be made from purified ploy-A-containing RNA (Muglia and Locker, unpublished results). In the present experiments, we found it useful to quantitate intact mRNA as well as total hybridization (see below). 


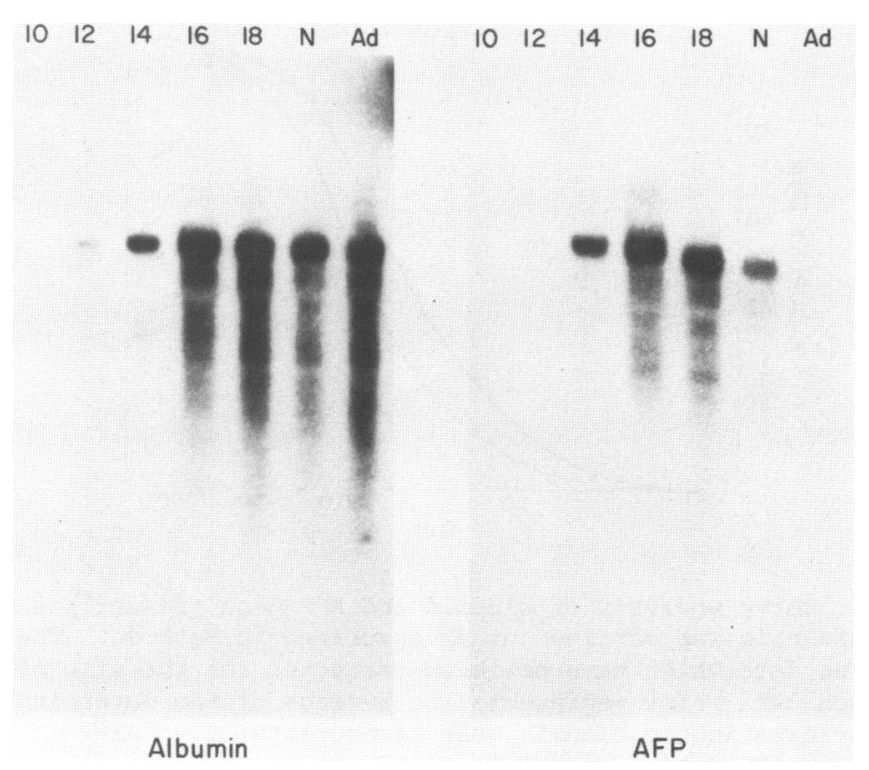

Figure 2. Detection of albumin and AFP mRNA in liver RNA. $10 \mu \mathrm{g}$ of RNA from 10-, 12-, 14-, 16-, and 18-day fetal livers, 1-day neonatal $(=22$ days gestation), and adult livers was analyzed as in Figure 1. The stained gels, not illustrated, appeared identical to the gel in Figure 1. The exposure is much shorter than that shown in Figure 1. However, hybridizations could not be detected in either 10-day lane, or in the adult liver AFP lane, at long exposures.

Each mRNA appears as one main hybridization band in short exposures. Albumin and AFP mRNA each have apparent molecular weights of $2.6 \mathrm{~kb}$ in this partially denaturing gel system (17), although the molecular weights on completely denaturing gels have been reported to be 2.27 and $2.24 \mathrm{~kb}$, respectively (25). Long exposures (e.g. Figure 1) demonstrate an additional hybridization band of $5.2 \mathrm{~kb}$ apparent molecular weight. This band disappears if the RNA is disaggregated by heating before loading on the gel (not shown). The $5.2 \mathrm{~kb}$ band thus appears to represent a specific aggregate rather than a discrete transcript.

mRNA Quantitation. Our blot-hybridizations used a number of probes that hybridize with different efficiencies. To calibrate the absolute levels of mRNA in these experiments, we carried out $R_{0} t$ curve analysis (Figure 3) of 18-day liver RNA, which contains high levels of both AFP and albumin mRNA. Using the $1160 \mathrm{bp}$ cDNA insert of pRSA57 and the $730 \mathrm{bp}$ insert of PRAF87 DNA, we determined $R_{0} t_{\frac{1}{2}}$ values of $2.5 \pm 0.3 \mathrm{Mole} \mathrm{sec} / 1$ for albumin and $2.8 \pm 0.3$ Mole sec/1 for AFP, respectively. Nahon et al. (9) 


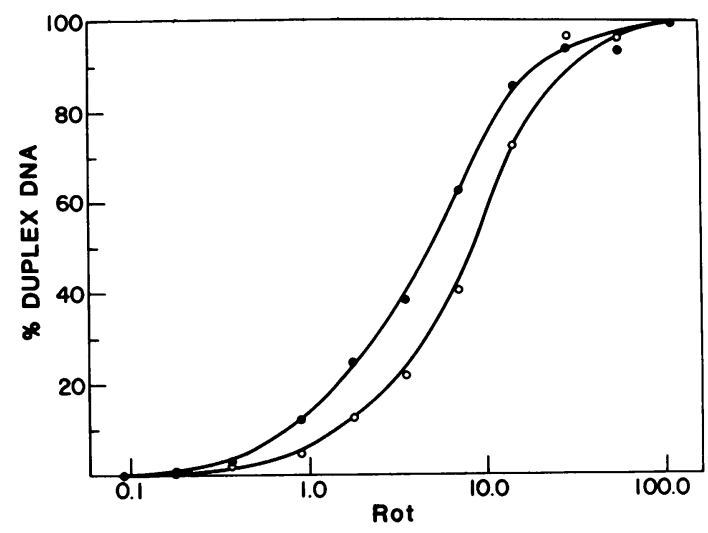

Figure 3. R $t$ curve analysis of albumin and AFP gene transcripts in 18-day liver RNA. Analysis was carried out as described in Methods. The plot illustrates raw data which have not been corrected for the size of the DNA fragment. Each data point represents the average of two determinations. - pRSA57 hybridization to albumin gene transcripts; 0, pRAF87 hybridization to AFP gene transcripts.

have reported a very similar $R_{0} t_{\frac{1}{2}}$ value of 2.05 for albumin mRNA in adult liver RNA. To calculate the number of molecules per cell, we used the experimentally determined $R_{0} t_{\frac{1}{2}}$ values of $1.5 \times 10^{-3}$ and $1.35 \times 10^{-3}$ for purified albumin and AFP mRNA, respectively, determined by Nahon et al. (9). We also calculated $32 \mathrm{pg}$ RNA/cell, based on the total RNA extracted per $\mathrm{ml}$ of tissue and the $259 \times 10^{6}$ nuclei per $\mathrm{ml}$ of rat liver reported by Weibel et al. (26).

All blot-hybridizations were analyzed densitometrically at several exposures, and the intensities were then normalized by the values obtained from the 18-day $R_{0} t_{\frac{1}{2}}$ values. These quantitative results are presented in Figure 4. Because we have not resolved the significance of the degraded RNA, we quantitated the data in two different ways, as total hybridizable RNA (Figure 4a) or intact mRNA (Figure 4b).

AFP and albumin mRNA were not detectable in the 10-day RNA preparation taken from the foregut region where the liver arises, even when very long exposure times were used. At maximum film exposure, we have calculated that this series of blots had a lower limit of detection of about 50 mRNA copies/cell (13). Both species are present in 12-day liver RNA; AFP mRNA reaches a peak level at 16 days and then declines, while albumin mRNA peaks at 18 days and persists through adulthood at comparable levels. In the yolk sac, both albumin and AFP mRNA are detectable at 10 days; AFP mRNA 

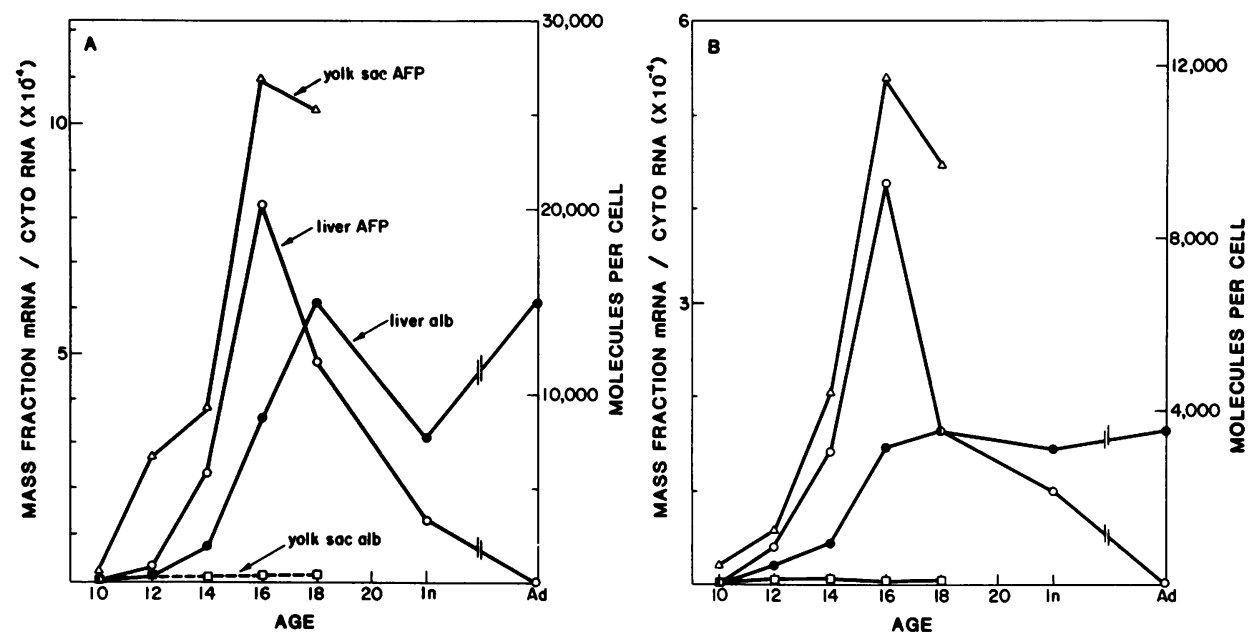

Figure 4. Quantitation of albumin and AFP gene transcripts. The autoradiograms were analyzed densitometrically, and the values were corrected for exposure time and normalized to the $R_{t} t_{1}$ values for 18-day liver RNA. Panel A shows the total hybridization to all species, while Panel $B$ shows the hybridization data for the specific mRNA bands alone. $\Delta$, yolk sac AFP; $\bullet$, liver AFP; 0 , liver albumin; $\square$, yolk sac albumin.

rises to a peak at 16 days, and albumin mRNA, though present at much lower levels, appears to do the same.

DNA methylation. We have previously analyzed the DNA methylation of the albumin and AFP genes in fetal, neonatal, and adult liver (5). The yolk sac shows a unique pattern of expression of these genes, where AFP mRNA is present at maximal levels while albumin mRNA levels are detectable but minimal; i.e., this pattern of expression is the reciprocal of the adult liver pattern. We therefore examined the DNA methylation of these genes in 18-day yolk sac. As in our previous studies, $10 \mu \mathrm{g}$ of DNA was digested with HpaII or MspI, resolved on agarose gels, blotted to nitrocellulose, and hybridized to probes from different regions of the two genes. The results of this analysis are shown in Figure 5. Unlike the liver at any stage, the yolk sac AFP gene is uniformly unmethylated, as evidenced by the fact that complete digest bands are present in the same relative proportions in the HpaII and the MspI hybridization patterns. The small amount of AFP gene methylation, detected as higher molecular weight partial digest bands, is probably accounted for by the stromal cells that are present. Conversely, the albumin gene is heavily methylated throughout its length. The level of methylation is greater than that of liver DNA at 


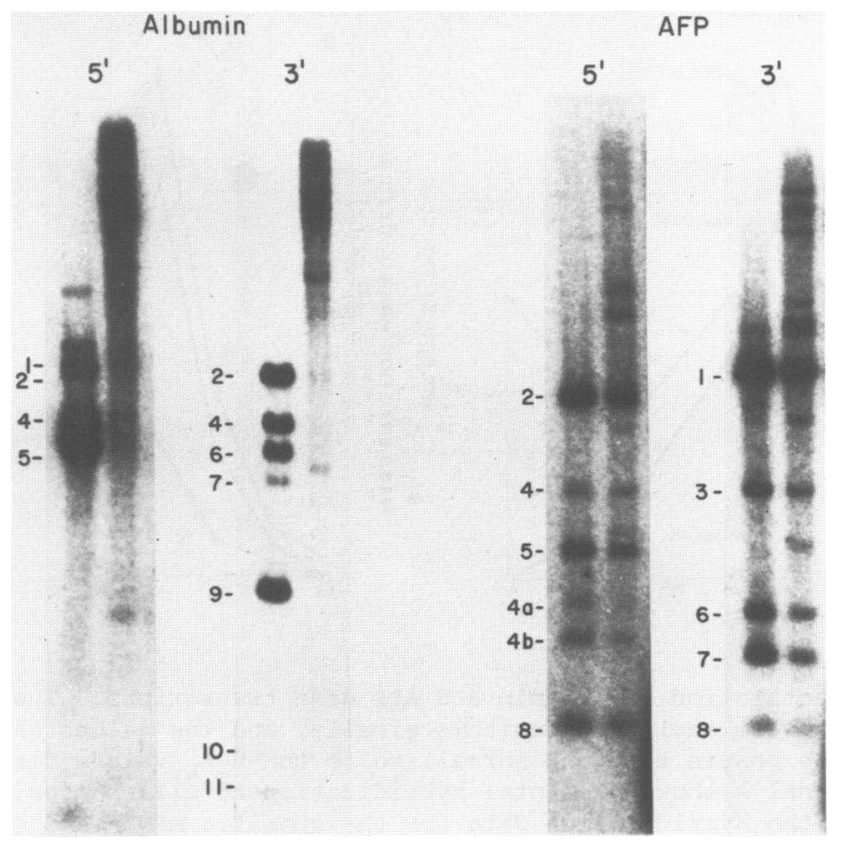

Figure 5. Southern blot analysis of methylation in yolk sac DNA. Each panel shows a matched pair of MspI (left) and HpaII (right) digests of yolk sac DNA. The albumin $5^{\prime}$ probe is pRSA57, and the albumin $3^{\prime}$ probe is pRSA13. The AFP 5' probe is pRAF87, and the AFP $3^{\prime}$ probe is pRAF65. These hybridization patterns should be compared to the data and restriction enzyme maps in Kunnath and Locker (5). The bands are numbered for each gene in order of decreasing size, except that the pooled yolk sac DNA preparations were heterozygous for an additional HpaII/MspI site that cuts $\mathrm{AFP}$ band 4 into $4 \mathrm{a}$ and $4 \mathrm{~b}$ (Locker, unpublished results).

any developmental stage, but somewhat lower than kidney or brain DNA (see Kunnath and Locker [5] for comparative data). This degree of methylation is especially surprising considering that yolk sac DNA is much less methylated than somatic DNA. Methylation within the albumin gene ranges from $75 \%$ to 1008 at different sites, while rat yolk sac DNA averages only 238 DNA methylation. This value was calculated from the number average molecular weights $\left(M_{n}\right)$ of MspI and HpaII digests of yolk sac DNA, which we determined by numerical analysis of densitometric scans of the digests (21). The hybridization patterns indicate that there is somewhat less methylation at several sites in the 3'-half of the albumin gene; these relatively undermethylated sites are not present in fetal or adult brain or adult kidney DNA (5). 


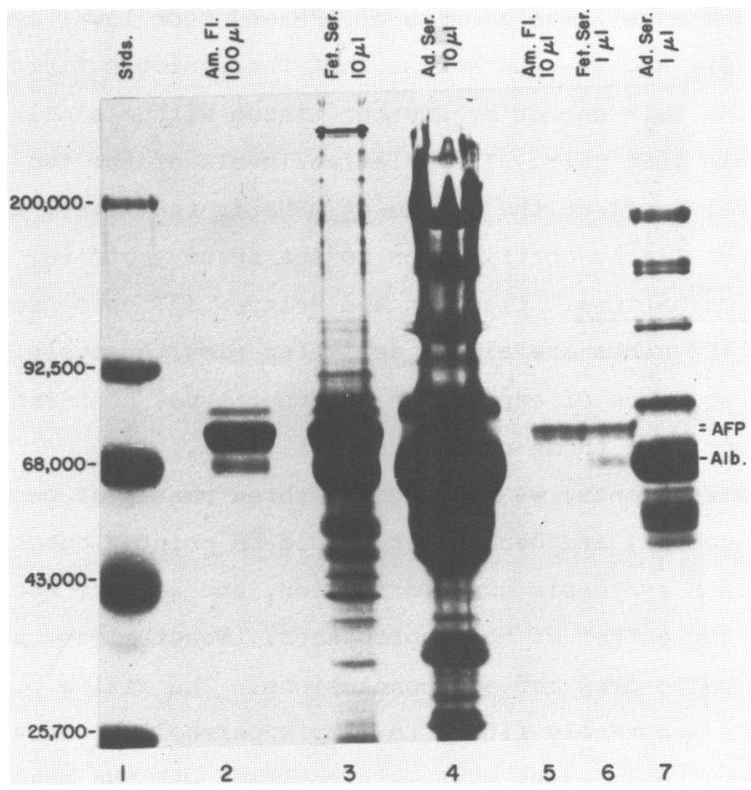

Figure 6. Electrophoretic analysis of serum and amniotic fluid proteins. Serum from 16-day fetuses and adults was purified from whole blood by centrifugation, and diluted into 28 sodium dodecyl sulfate, 58

B-mercaptoethanol, 8M urea. Amniotic fluid proteins were precipitated with acetone and redissolved in the same solution. Samples were resolved on a 7.58 acrylamide-SDS gel and stained with Coomassie blue. The information above the figure indicates the relative volume of each fluid specimen resolved in the gel lane. Each sample is shown at two concentrations to allow discrimination of specific bands and also to illustrate the relative concentrations of proteins. It should be noted that although the protein content of amniotic fluid is much lower than serum, there is a great deal more of it in the conceptus. Also, note that mature serum not only lacks AFP but has a much higher concentration of albumin and other serum proteins than fetal serum. This is accounted for, not by a higher content of mRNA per cell, but by the much larger relative size of the adult liver.

\section{DISCUSSION}

Modes of coordinate gene expression. Our data indicate three active modes of expression for the albumin-AFP gene pair in the rat. Adult liver cells synthesize a great deal of albumin and very little AFP. Fetal liver cells synthesize large amounts of both. Yolk sac cells synthesize large amounts of AFP and a small amount of albumin. For completeness, tissues such as brain can be considered in a fourth, nonexpressing, mode. The first two modes are clearly reflected in the adult and fetal levels of serum proteins (Figure 6): adult serum contains only albumin, while fetal serum contains high levels of both proteins. We have observed that 
amniotic fluid contains large amounts of AFP and much lower amounts of albumin (Figure 6), and we hypothesize that the amniotic fluid proteins are synthesized by the yolk sac or by another tissue with a similar mode of expression of this gene pair. The relative levels of the two proteins in fetal serum closely reflect the levels of mRNA in the liver, and the yolk sac probably makes little contribution to the serum proteins.

Regulatory mechanisms. Tilghman and Belayew (7) have proposed that the albumin and AFP genes are always activated simultaneously. Our analysis of the sequence of expression in two tissues confirms and extends their observations, and those of sellem et al. (8).

From our measurements, we distinguish three phases of gene activity: induction, enhancement, and decay. It should be pointed out that mRNA levels reflect both synthesis and degradation, and we have not carried out specific assays for either of these-processes. Nonetheless, analysis of our data allows us to draw certain conclusions. The rising portions of the curves all can be accurately fitted to simple parabolas (concave upward). If the rate of synthesis of an mRNA were constant, in the absence of turnover the accumulation would be linear. Any turnover would make the curve concave downward. Therefore, the curves that we observe must result from increasing synthesis (enhancement) regardless of the rate of turnover. Extrapolation of these parabolas indicates that albumin and AFP mRNA synthesis begin concurrently on about day 11 in the liver and day 9 in the yolk sac.

In liver, though, their gene induction is virtually simultaneous, the albumin and AFP mRNA levels do not rise strictly in parallel. AFP peaks at 16 days, while albumin does not peak until 18 days. The yolk sac AFP mRNA peak is also at 16 days. In the liver, following their 16-day peak, the AFP mRNA levels fit a curve of exponential decay (correlation coefficient = $0.997)$ with a half life of 2.2 days. This decay is not simply turnover of the RNA, since AFP transcription is still taking place $(7,27)$. Rather, the decay reflects a decline in the rate of synthesis.

On the basis of these observations, we can propose a model for the coordinate expression of albumin and AFP (1.) Induction: The albumin and AFP genes appear to be activated simultaneously in liver and in yolk sac, as proposed by Tilghman and Belayew. However, activation results only in a low basal level of synthesis. (2.) Enhancement: The parabolic rise in the level of an mRNA reflects enhancement, probably through synthesis of a specific transcription factor. Although enhancement could occur by other 
mechanisms, such as stabilization of mRNA or an increase in the proportion of cells synthesizing the mRNA, Nahon et al. (9) have presented evidence for the lack of post-transcriptional control of these genes, and our histologic immunoperoxidase studies (Locker, unpublished results) show no change in the proportion of liver and yolk sac cells synthesizing albumin and AFP during enhancement. In yolk sac, only the AFP gene is enhanced, while in liver both genes are enhanced although with somewhat different timing during fetal development. The two genes are probably enhanced by different factors. (3.) Decay: We hypothesize that the exponential decay of AFP mRNA synthesis in liver occurs when synthesis of the AFP specific enhancing factor decreases; that the factor that has a 2.2 day half life; and that the albumin-gene-enhancing transcription factor is synthesized constitutively in liver after its initial induction.

DNA methylation and transcription control. The new data reported in this paper on the yolk sac genes should be considered together with our previous analysis of albumin and AFP gene methylation in developing liver and control tissues (5). In that paper, we showed that the albumin and AFP genes had intermediate (higher than adult liver but lower than kidney or brain) levels of methylation in fetal liver, and that both genes decreased in methylation by adulthood. The albumin gene became uniformly demethylated and remained active, while the AFP gene became focally demethylated at two sites in the $3^{\prime}$-half of the gene. The yolk sac albumin and AFP genes are methylated in yet another unique pattern. It is difficult to correlate all of these states with a transcription control mechanism based on gene methylation. Nonetheless, all are unique stage-specific patterns that can be readily identified. The yolk sac albumin gene is particularly remarkable, in that it is hypermethylated in a tissue which is deficient in DNA methylation. These changes thus parallel the changes in tissue expression, and we cannot rule out that methylation has an important role in the enhancement process. Nonetheless, we think it is unlikely that these methylation patterns actively control transcription. They could reflect the accessibility of regions of the genes to methylases or the constitutive levels of the methylases.

\section{ACKNOWLEDGEMENTS}

This work was supported by National Institutes of Health Grant GM27795. L.M. was supported by the Medical Scientist Training Program, GM07281. 
*Present address: School of Medicine, Department of Pathology, University of Pittsburgh, 777A Scaife Hall, Pittsburgh, PA 15261, USA

+ To whom correspondence should be sent at his present address

\section{REFERENCES}

1. Gorin, M.B., Cooper, D.L., Eiferman, F., van de Rijn, P., and Tilghman, S.M. (1981) J. Biol. Chem. 256, 1954-1959

2. Jagodzinski, L.L., Sargent, T.D., Yang, M., Glackin, C., and Bonner, J. (1981) Proc. Nat. Acad. Sci., USA 78, 3521-3525

3. Ohno, S. (1981) Proc. Nat. Acad. Sci., USA 78, 7657-7661

4. Ingram, R.S., Scott, R.W., and Tilghman, S.M. (1981) Proc. Nat. Acad. Sci., USA 78, 4694-4698

5. Kunnath, L., and Locker, J. (1983) EMBO J. 2, 317-324

6. Kunnath, L., and I.ocker, J. (1984) Submitted for publication

7. Tilghman, S.M., and Belayew, A. (1982) Proc. Nat. Acad. Sci., USA $79,5254-5257$

8. Sellem, C.H., Frain, M., Erdos, T., and Sala-Trepat, J.M. (1984) Devel. Biol. 102, 51-60

9. Nahon, J.L., Gal, A., Frain, M., Sell, S., and Sala-Trepat, J.M. (1982) Nucleic Acids Res. 10, 1895-1911

10. Beaudoin, A.R. (1980) In The Laboratory Rat, Vol. II, Baker, H.J.., Lindsey, J.R., and Weisbroth, S.H., eds. New York, Academic Press, pp. 75-103

11. Sargent, T.D., Wu, J.R., Sala-Trepat, J.M., Wallace, R.B., Reyes, A.R., and Bonner, J. (1979) Proc. Nat. Acad. Sci., USA 76, 3256-3260

12. Sargent, T.D., Yang, M., and Bonner, J. (1981) Proc. Nat. Acad. Sci., USA 78, 243-246

13. Muglia, L., and Locker, J. (1984) Proc. Nat. Acad. Sci., USA 81, 3635-3639

14. Noyes, B.E., Mevarech, M., Stein, R., and Agarwal, K.L.

Proc. Nat. Acad. Sci., USA 76, 1170-1774

15. Chirgwin, J.M., Przybyla, A.E., MacDonald, R.J., and Rutter, W.E. (1979) Biochemistry 18, 5294-5299

16. Volkin, E., and Cohn, W.E. (1954) In Methods of Biochemical Analysis, Vol. 1, Glick, D. ed. Interscience, New York pp. 287-306

17. Locker, J. (1979) Anal. Biochem. 98, 358-367

18. Tse, T.P.H., Morris, H.P., and Taylor, J.M. (1978) Biochemistry 17, 3121-3128

19. Rigby, P.W.J., Dieckmann, M., Rhodes, C., and Berg, P. (1977) J. Mol. Biol. 113, 237-251

20. Locker, J. (1981) In Electrophoresis '81 Allen, R.C. and Arnaud, P., eds. deGruyter, Berlin pp. 617-625

21. Kunnath, L., and Locker, J. (1982) Biochem. Biophys. Acta 699, 264-271

22. Southern, E.M. (1975) J. Mol. Biol. 98, 503-517

23. Kunnath, L., and Locker, J. (1982) Nucleic Acids Res. 10, 3877-3892

24. Hames, B.D. (1981) In Gel Electrophoresis of Proteins: A Practical Approach Hames, B.D. and Rickwood, D., eds. IRL Press, London pp. $1-91$

25. Sala-Trepat, J.M., Dever, J., Sargent, T.D., Thomas, K., Sell, D., and Bonner, J. (1979) Biochemistry 18, 2167-2178

26. Weibel, E.R., Staübli, W., Rudolf, H.,Gnägi, R., and Hess, F.A. (1969) J. Cell. Biol. 42, 68-90

27. Chiu, J.F., Massari, R.J., Schwartz, C.E., Meisler, N.T., and Thanassi, J.W. (1981) Nucleic Acids Res. 9, 6917-6933 